\title{
Postnatal Changes in Functional Activities of the Pig's Brain: A Combined Functional Magnetic Resonance Imaging and Immunohistochemical Study
}

\author{
Marong Fang ${ }^{a}$ Dietrich E. Lorke ${ }^{\mathrm{c}}$ Jicheng $\mathrm{Li}^{\mathrm{a}}$ Xiangyang Gong ${ }^{\mathrm{b}}$ \\ Jason C.C. Yew ${ }^{d}$ D.T. Yew ${ }^{d}$ \\ ${ }^{a}$ Department of Anatomy, Medical College, and ${ }^{b}$ Department of Radiology, Zhejiang University, \\ Hangzhou, China; ' Institute of Anatomy II, University Hospital Hamburg Eppendorf, Hamburg, Germany; \\ ${ }^{\mathrm{d}}$ Department of Anatomy, Chinese University of Hong Kong, SAR, China
}

\section{Key Words}

Development · Functional activation · GABA · Pain •

Passive movement

\begin{abstract}
Developmental changes in brain activation after pain stimulation and after passive movement of the hind paw were assessed by functional magnetic resonance imaging (fMRI) in pigs of postnatal ages 2, 4 and 6 months. Response patterns were correlated with histological maturation parameters. At 2 months, fMRI failed to detect brain activation after pain stimulation and revealed weak, but widespread activation after passive movement. At 4 months, strong reaction of numerous cortical areas on the contralateral side was seen after pain stimulation. Following passive movement, activation was weaker but more widespread, and the brainstem was also involved. By 6 months, cortical activation became more restricted to the contralateral sensory cortex and brainstem after pain stimulation and to the contralateral sensory and ipsilateral premotor and motor cortices after passive movement. Neocortical synaptophysin immunoreaction increased significantly between 2 and 4 months and slightly decreased by 6 months. The density of GABA-immunoreactive neurons and fibers sig-
\end{abstract}

nificantly increased, reaching a maximum at 6 months. Our studies indicate that remodeling of synapses and development of inhibitory GABA neurons last until 6 months postnatally, when the fMRI response of the pig's brain also attains its mature adult pattern.

Copyright $(2005$ S. Karger AG, Basel

\section{Introduction}

The pig's brain lends itself as a useful model in medical research, due to its similarities with the human brain [1-3]. It is gyrencephalic, and its overall shape, gyral pattern as well as grey/white matter distribution are similar to those of the human brain [4]. Due to its size, its activity can be relatively easily examined by magnetic resonance imaging (MRI). For these reasons, the pig brain has recently been the subject of numerous studies monitoring the effect of different CNS lesion models by MRI [3, 5, 6]. During development, the pig CNS also shows striking parallels to that of the human. The growth spurt extends from late prenatal to early postnatal life, and changes in brain morphology and the sequence of nervous system maturational changes are comparable for pigs and humans $[3,4]$. Hence it is also an appropriate model for traumatic injury of the developing brain frequently ex-

\section{KARGER}

Fax +4161306 1234 E-Mail karger@karger.ch www.karger.com
(C) 2005 S. Karger AG, Basel

1424-862X/05/0145-0222\$22.00/0

Accessible online at:

www.karger.com/nsg
D.T. Yew

G15 Choh-Ming Li Basic Medical Sciences Building

Chinese University of Hong Kong, Shatin

Hong Kong SAR (China)

Tel. +852 2609 6899, Fax +852 2603 5031, E-Mail david-yew@cuhk.edu.hk 
amined by MRI $[4,7,8]$. All these studies, including those on un-lesioned pig brains $[9,10]$, have been performed in anesthetized animals. In contrast, there is remarkable scarcity in information on functional MRI (fMRI) responses in conscious pigs, allowing to relate structure to function [11]. However, significant changes in fMRI signal intensity have been described during anesthesia [12]. In addition, a systematic analysis of fMRI responses in the developing pig brain is lacking. There are no data correlating maturation parameters during the postnatal development of the pig brain with its functional activity [13]. We have therefore evaluated the fMRI responses of the brains of un-anesthetized young pigs (2-6 months postnatally) upon pain stimulation and initiation of passive movement. We have then correlated these data with the cytochemical brain development of these animals, i.e. immunoreaction for synaptophysin, a synaptic vesicle protein [14], and for GABA, the most prominent inhibitory neurotransmitter in the brain, which plays a major role in neuronal plasticity [15].

\section{Materials and Methods}

\section{Animal Care}

Fifteen female Bama minipigs (weight $2.5-17 \mathrm{~kg}$ ) were obtained from the Laboratory Animal Center for minipigs in the Guangxi Medical University, China, $2(\mathrm{n}=4), 4(\mathrm{n}=5)$ and 6 months $(\mathrm{n}=$ $6)$ post partum. The pigs were housed in large cages (1 pig/cage) and were maintained at $22-24^{\circ} \mathrm{C}$ on a 12 -hour light:12-hour dark cycle. All care was in accordance with the guidelines issued by the Animal Use and Care Committee in the Chinese University of Hong Kong and with the guidelines published in the NIH Guide for the Care and Use of Laboratory Animals. The procedure for immobilization and functional brain imaging in fully conscious animals was approved and monitored by the Ethical Committee of the University and assisted by the technicians in the Laboratory Animal Center.

\section{Pre-Experiment Preparation}

For maximal immobilization and exact positioning in brain fMRI studies, a special custom-built holder for the pig's body and head was made of Plexiglas material. The pigs were restrained in this holder in a supine position and further fastened by elastic bands. An additional foam headrest was made to restrain the head from movement in any direction, resulting in optimal fixation of the head and minimal motion during fMRI. Un-anesthetized pigs were used to map the neural activation of swine brain upon (1) pain stimulation and (2) passive movement of the hind paw.

\section{Stimulation Procedures}

Prior to scanning, each pig was trained to adapt to the restraint in the custom-built holder, the MRI machine and the noise. All tasks were performed by the same experimenter, who was visually directed by a multimedia projector. The operator was instructed to initiate the painful stimuli or to start the passive movement, when he saw 'do' on the screen, and not to give any performance, when he saw 'rest' on the screen. In the painful stimulation task, the experimenter stimulated the medial side of the right hind paw with a sharp plastic pin at a rhythm of 60 times per minute. Block design was selected and task periods were repeated for 8 times in each experiment with a 20 -second rest followed by 20 -second epochs of stimulation. Total time for this task and scanning was $320 \mathrm{~s}$. In the passive movement task, the task was a repeated flexion-extension movement of the knee joint of the pig's right hind paw performed by the technician, while the proximal part of the leg was immobilized by elastic bands. Brisk passive movement was elicited every second. Experiment time was the same as for pain stimulation.

\section{fMRI Acquisition}

Images were acquired on a 1.5-tesla unit (MAGNETOM Sonata; Siemens, Erlangen, Germany) with a CP head array coil. Imaging started after $30 \mathrm{~min}$ of adaptation, began with axial spin-echo (SE) $\mathrm{T}_{1}$-weighted imaging $(\mathrm{TR} / \mathrm{TE}=500 \mathrm{~ms} / 15 \mathrm{~ms}$, slice thickness/ gap $=4 \mathrm{~mm} / 1 \mathrm{~mm}$, field of view, FOV $=180 \times 180 \mathrm{~mm}$, acquisition matrix $=256 \times 256,2$ acquisitions and 12 slices) parallel to the intercommissural line covering the whole brain, and three-dimensional gradient-echo imaging $(\mathrm{TR} / \mathrm{TE}=40 \mathrm{~ms} / 6 \mathrm{~ms}, \mathrm{FOV}=$ $180 \times 180 \mathrm{~mm}$, flip angle $=35^{\circ}$, acquisition matrix $=256 \times 205$, 2 acquisitions and 60 slices) in the sagittal plane. Functional areas were localized according to a previously published stereotactic atlas of the pig brain [16]. $\mathrm{T}_{2}$-sensitive, gradient-echo planar images were obtained with the following parameters: TR/TE $=2,000 \mathrm{~ms} / 60 \mathrm{~ms}$, flip angle $90^{\circ}$, slice thickness $/$ gap $=4 \mathrm{~mm} / 1 \mathrm{~mm}$, matrix $64 \times 64$ and $\mathrm{FOV}=180 \times 180 \mathrm{~mm}$; scan time for each run was $2 \mathrm{~s}$. Twelve contiguous axial images were acquired with the same slice to the $\mathrm{T}_{1}$-weighted imaging. The onset of fMRI collection was synchronized with stimulus presentation.

\section{Statistical Analysis}

The functional images were analyzed off-line with the Analysis of Functional NeuroImages (AFNI, http://afni.nimh.nih.gov/). Functional images were resliced and smoothed with a Gaussian spatial filter of $5 \mathrm{~mm}$ full width at half maximum; motion correction was also performed. The realignment of the EPI images and coregistration of the EPI images with the $T_{1}$-weighted images were carried out. The first four images from each run were discarded. Correlation analysis was performed to test the correlation between MRI signal changes of pig's brain and stimulation patterns. A t test statistical map was yielded and superimposed onto $\mathrm{T}_{1}$-weighted images. A statistical threshold was set at the height threshold of $\mathrm{p}<0.01$. When the motion distance of an individual animal was larger than 3 voxel size, all the results were discarded and the fMRI performed again in another 5 days.

\section{Immunofluorescence}

At the conclusion of fMRI, 4 pigs of each of the three age groups $(2,4,6$ months of age) were killed with an overdose of sodium pentobarbital (1 g/kg body weight, i.p.). The porcine brains were dissected out quickly and tissue blocks $\left(1 \times 1 \times 1 \mathrm{~cm}^{3}\right)$ of the sensory cortex were immersion fixed in $4 \%$ paraformaldehyde for $15 \mathrm{~h}$. Specimens were then cryoprotected in $30 \%$ sucrose at $4^{\circ} \mathrm{C}$, rapidly frozen, sectioned at $40 \mu \mathrm{m}$ using a cryomicrotome, collected in PBS and processed as free-floating sections. After brief PBS rinses, sections were incubated for $30 \mathrm{~min}$ at room temperature in a blocking solution of $1 \%$ normal donkey serum in PBS with $0.3 \%$ Triton X-100, 

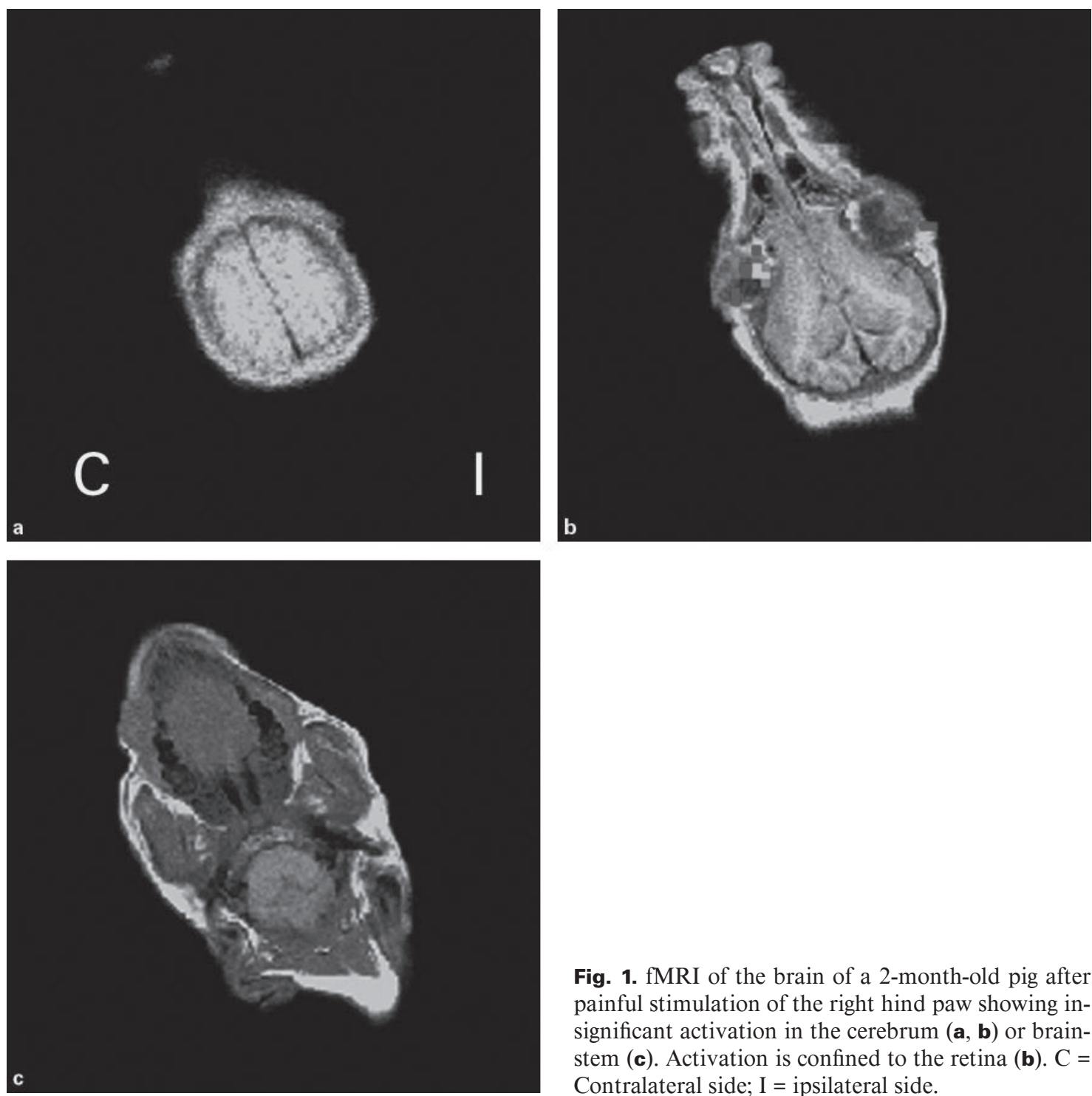

Fig. 1. fMRI of the brain of a 2-month-old pig after painful stimulation of the right hind paw showing insignificant activation in the cerebrum $(\mathbf{a}, \mathbf{b})$ or brainstem (c). Activation is confined to the retina (b). $\mathrm{C}=$ Contralateral side; $\mathrm{I}$ = ipsilateral side.

and were then incubated overnight at room temperature in the following primary antisera: polyclonal rabbit antibody against GABA (1:300 dilution; Chemicon, Temecula, Calif., USA) and monoclonal mouse antibody against synaptophysin (1:150 dilution; Sigma, St. Louis, Mo., USA). After this incubation, sections were rinsed three times for 10 min each in PBS and incubated in the fluorescent secondary antibody solution (donkey anti-rabbit IgG, FITC, Molecular Probes, Eugene, Oreg., USA; donkey anti-mouse IgG, FITC, Jackson Immuno Research, West Grove, Pa., USA) for $2 \mathrm{~h}$ at room temperature, applied at a dilution of 1:300, respectively.

After washing three times for 10 min each in PBS, the sections were finally mounted on gelatin-coated slides; coverslips were placed on slides using a water-soluble anti-fade mount medium (Dakopatts, Glostrup, Denmark). Control experiments were performed with the primary antisera omitted. No staining was observed under these conditions.

\section{Quantification of Immunofluorescent Staining}

Positively staining neurons and fibers were photographed $(\times 10$ objective) using a SPOT Cooled Color Digital Camera 32 (Diagnostic Instruments, Sterling Heights, Mich., USA) on an Axiophot 2 microscope (Carl Zeiss, Oberkochen, Germany) equipped for epifluorescence using 488-nm exciting wavelength. The number of GABA-positive neurons and the density of GABA-positive fibers were analyzed in twenty fields randomly selected in the sensory cortex [16] of each section, utilizing an image analysis software (Metamorph 4.0; Diagnostic Instruments). The same technique was applied to qualify the number of synaptophysin-immunoreactive fibers including terminals. Results for each age group were given as mean number of GABA-immunoreactive neurons per $700 \mu \mathrm{m}^{2}$, of GABA-immunoreactive fibers per $700 \mu \mathrm{m}^{2}$ and of synaptophysin-immunoreactive fibers or terminals per $700 \mu \mathrm{m}^{2}$. 

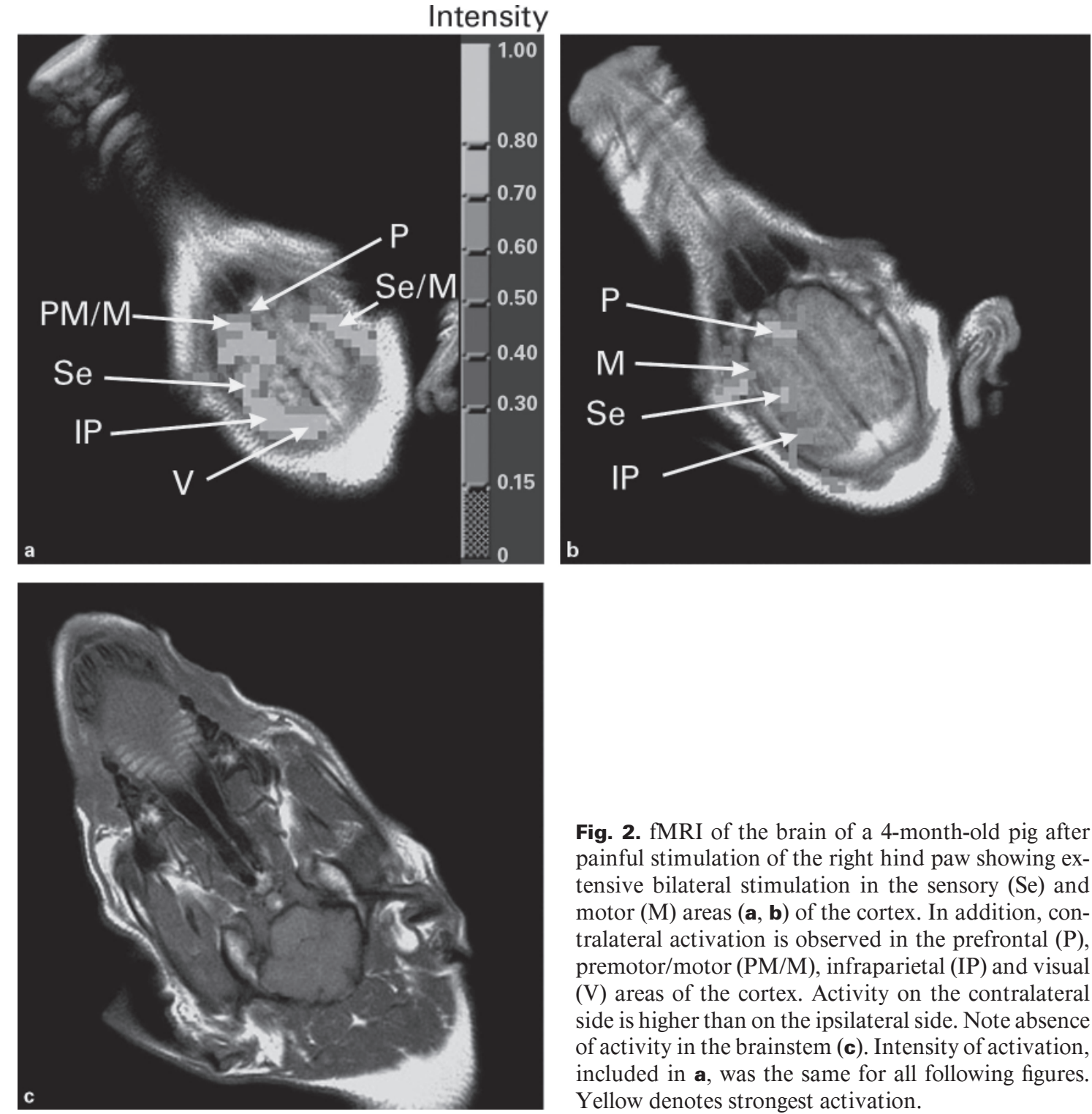

Fig. 2. fMRI of the brain of a 4-month-old pig after painful stimulation of the right hind paw showing extensive bilateral stimulation in the sensory $(\mathrm{Se})$ and motor $(\mathrm{M})$ areas $(\mathbf{a}, \mathbf{b})$ of the cortex. In addition, contralateral activation is observed in the prefrontal $(\mathrm{P})$, premotor/motor (PM/M), infraparietal (IP) and visual (V) areas of the cortex. Activity on the contralateral side is higher than on the ipsilateral side. Note absence of activity in the brainstem (c). Intensity of activation, included in $\mathbf{a}$, was the same for all following figures. Yellow denotes strongest activation.

In order to avoid counting of the same areas many times, the Neurolucida 2000 software (MicroBrightField, Colchester, Vt., USA) was used, which also recorded the areas counted on computer so that there was no duplication in the selection of sites. The counted numbers were also corrected with an Abercrombie formula morphometrically [17].

\section{Statistical Analysis}

One-way ANOVA was used to compare the intergroup differences in the mean numbers of immunofluorescent neurons and fibers among the three age groups. Results were considered statistically significant if $\mathrm{p}<0.05$. In all cases, the investigator responsible for counting was 'blinded' to the age status of each pig.

\section{Results}

Using our custom-built pig holder, fMRI studies of the brain of un-anesthetized pigs were relatively easy to perform. In all the functional runs, head motion was minimal, and pain simulation as well as passive movement of the hind paw resulted in a statistically significant fMRI response. During a period of up to $3 \mathrm{~h}$ of restraint, it was possible to acquire high-resolution anatomic as well as functional images, which were free of ostensible movement artifacts. Response or activation patterns were relatively uniform within each age group and highly reproducible. However, in older animals, slight interindividu- 

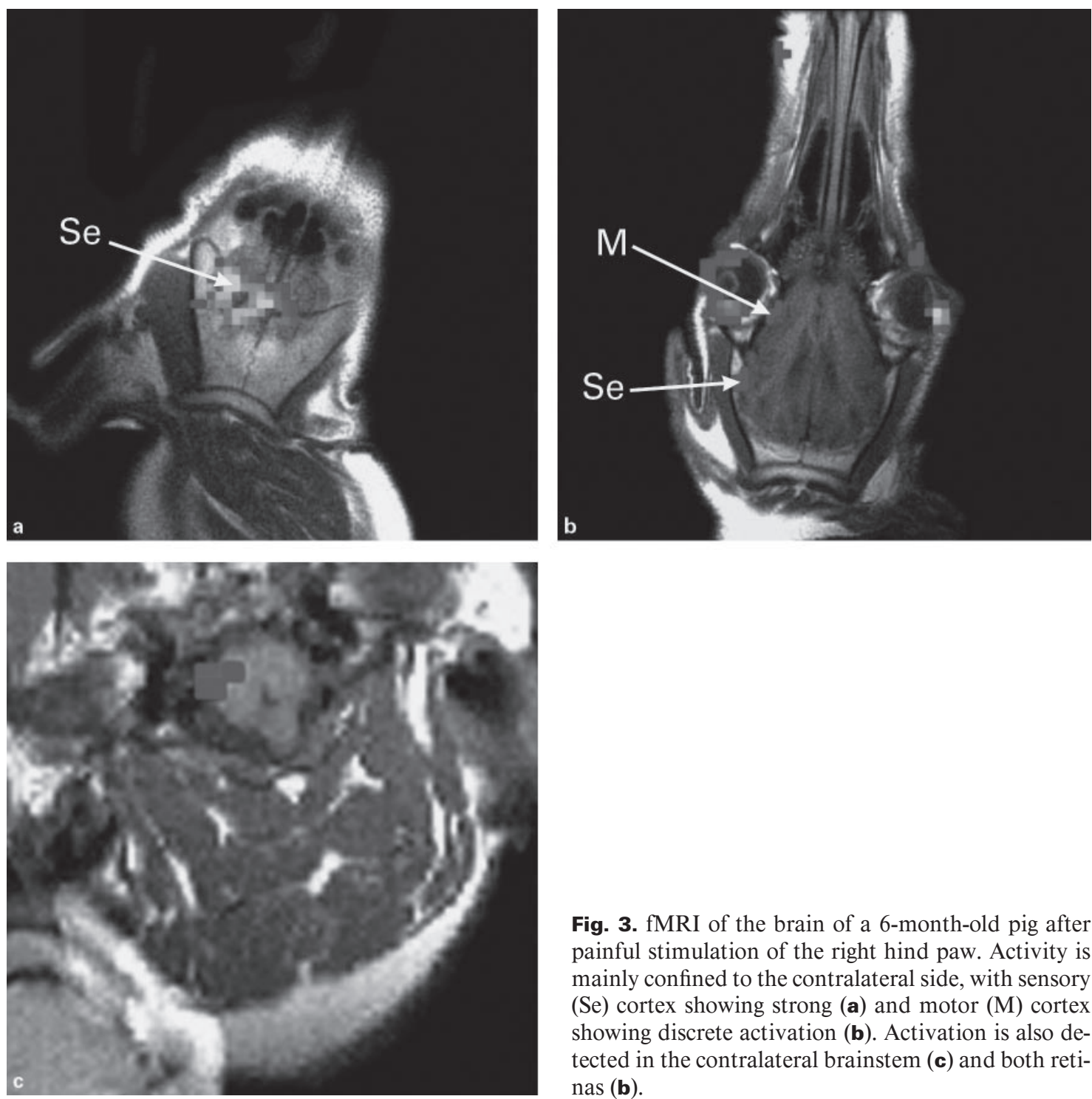

Fig. 3. fMRI of the brain of a 6-month-old pig after painful stimulation of the right hind paw. Activity is mainly confined to the contralateral side, with sensory (Se) cortex showing strong (a) and motor (M) cortex showing discrete activation (b). Activation is also detected in the contralateral brainstem (c) and both retinas (b).

al differences were observed in reaction intensity, but there was no variation in the activation pattern.

\section{Brain Activation upon Pain Stimulation}

The results listed below were representative activation maps from pigs of each age group. No significant deviation in activation sites was observed after comparing and matching fMRI activation sites recorded in pigs of the same age group. The representative activation maps therefore denoted the patterns of activation sites shown by the majority (at least $80 \%$ of the animals) of that age group. On the other hand, the activating sites were quite different between age groups. Upon painful stimulation, the relative overall proportions of the cortex activated (means $\pm \mathrm{SD}$ ), as computed from the images, were 5.56 $\pm 0.90 \%$ per animal at 2 months, $22.00 \pm 1.83 \%$ per animal at 4 months and $9.33 \pm 1.04 \%$ per animal at 6 months. $t$ test evaluation indicated significant differences between 4 months and either 2 or 6 months $(p<0.001)$, whilst that between 2 and 6 months was marginally significant ( $\mathrm{p}=0.05)$. This confirmed a stimulation-induced increase in activated sites at 4 months of age in the pig.

At the age of 2 months, painful stimulation of conscious pigs only resulted in very weak activation of the cortex or of deeper subcortical structures, which gave no significant signal concentration in the fMRI (fig. 1a-c). 

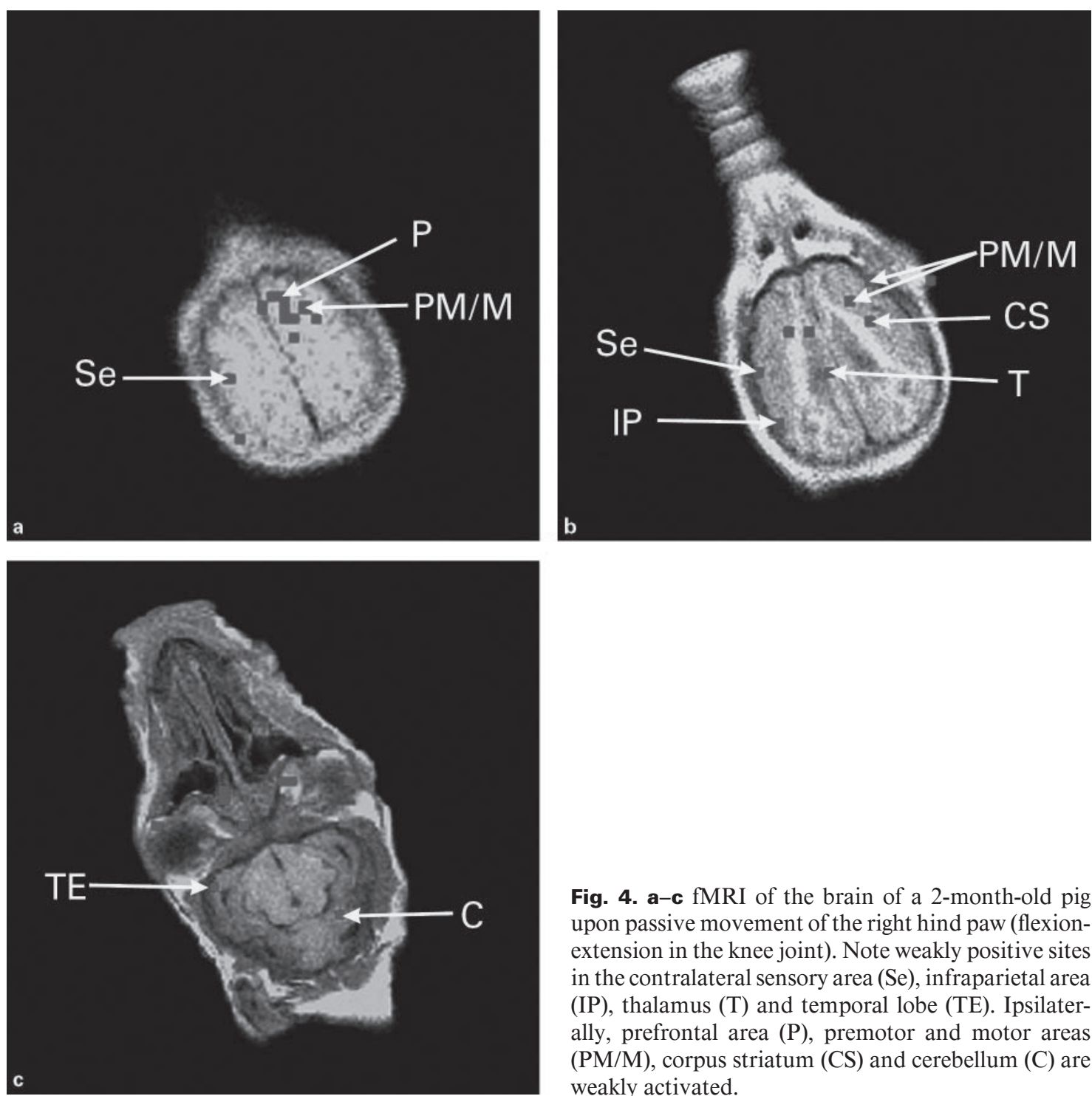

Fig. 4. a-c fMRI of the brain of a 2 -month-old pig upon passive movement of the right hind paw (flexionextension in the knee joint). Note weakly positive sites in the contralateral sensory area (Se), infraparietal area (IP), thalamus (T) and temporal lobe (TE). Ipsilaterally, prefrontal area $(\mathrm{P})$, premotor and motor areas $(\mathrm{PM} / \mathrm{M})$, corpus striatum (CS) and cerebellum (C) are weakly activated.

In 4-month-old animals, extensive activation sites were observed on both sides of the brain after pain stimuli. Activation was, however, higher and occupied a larger area on the contralateral side. Bilateral response was detected in the motor and sensory cortices, whereas activation was confined to the contralateral side in the prefrontal, premotor, infraparietal and visual cortices (fig. 2a, b). In all these brain regions, activation was relatively strong. In contrast, no functional response was found in the brainstem (fig. 2c).

At 6 months of age, fMRI response to pain stimuli was more discrete and restricted to smaller brain regions (fig. 3a-c). Activation was predominantly registered on the contralateral side, involving mainly the sensory cortex and a very small part of the motor area (fig. 3a, b). At this age, functionally active sites were also detected in the contralateral brainstem (fig. 3c).

\section{Brain Activation upon Passive Movement}

Already at the age of 2 months, passive movement of the pig's hind limb was associated with functional activation of multiple areas on both sides of the brain. fMRI response was, however, relatively weak (fig. $4 a-c)$. On the ipsilateral side, activation was observed in the prefrontal, premotor/motor cortices and the corpus striatum (fig. 4ac). In addition, an ipsilateral response was detected in the 

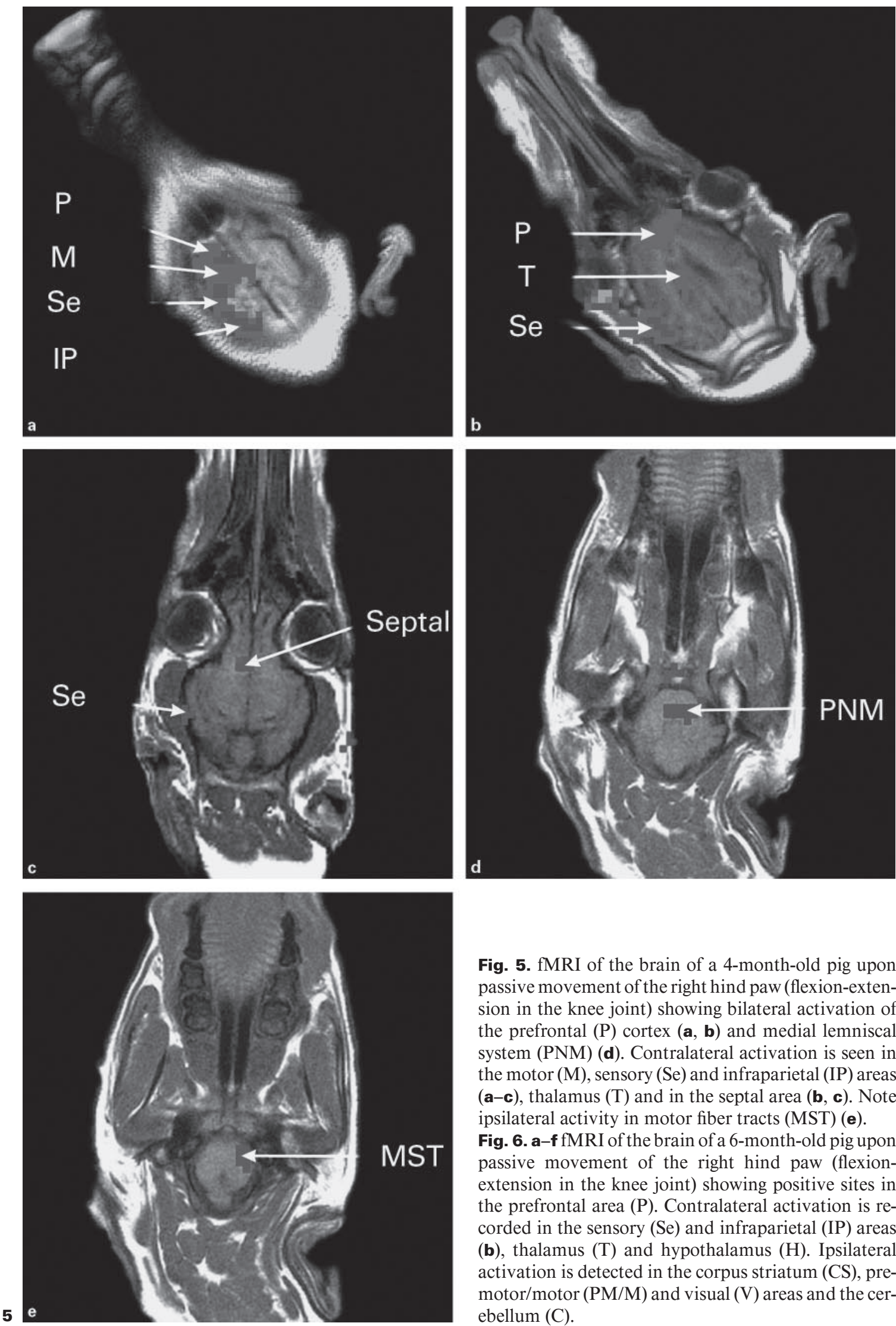

Fig. 5. fMRI of the brain of a 4-month-old pig upon passive movement of the right hind paw (flexion-extension in the knee joint) showing bilateral activation of the prefrontal $(\mathrm{P})$ cortex $(\mathbf{a}, \mathbf{b})$ and medial lemniscal system (PNM) (d). Contralateral activation is seen in the motor (M), sensory (Se) and infraparietal (IP) areas $(\mathbf{a}-\mathbf{c})$, thalamus $(\mathrm{T})$ and in the septal area $(\mathbf{b}, \mathbf{c})$. Note ipsilateral activity in motor fiber tracts (MST) (e).

Fig. 6. a-f fMRI of the brain of a 6-month-old pig upon passive movement of the right hind paw (flexionextension in the knee joint) showing positive sites in the prefrontal area (P). Contralateral activation is recorded in the sensory (Se) and infraparietal (IP) areas (b), thalamus $(\mathrm{T})$ and hypothalamus $(\mathrm{H})$. Ipsilateral activation is detected in the corpus striatum (CS), premotor/motor (PM/M) and visual (V) areas and the cerebellum (C). 

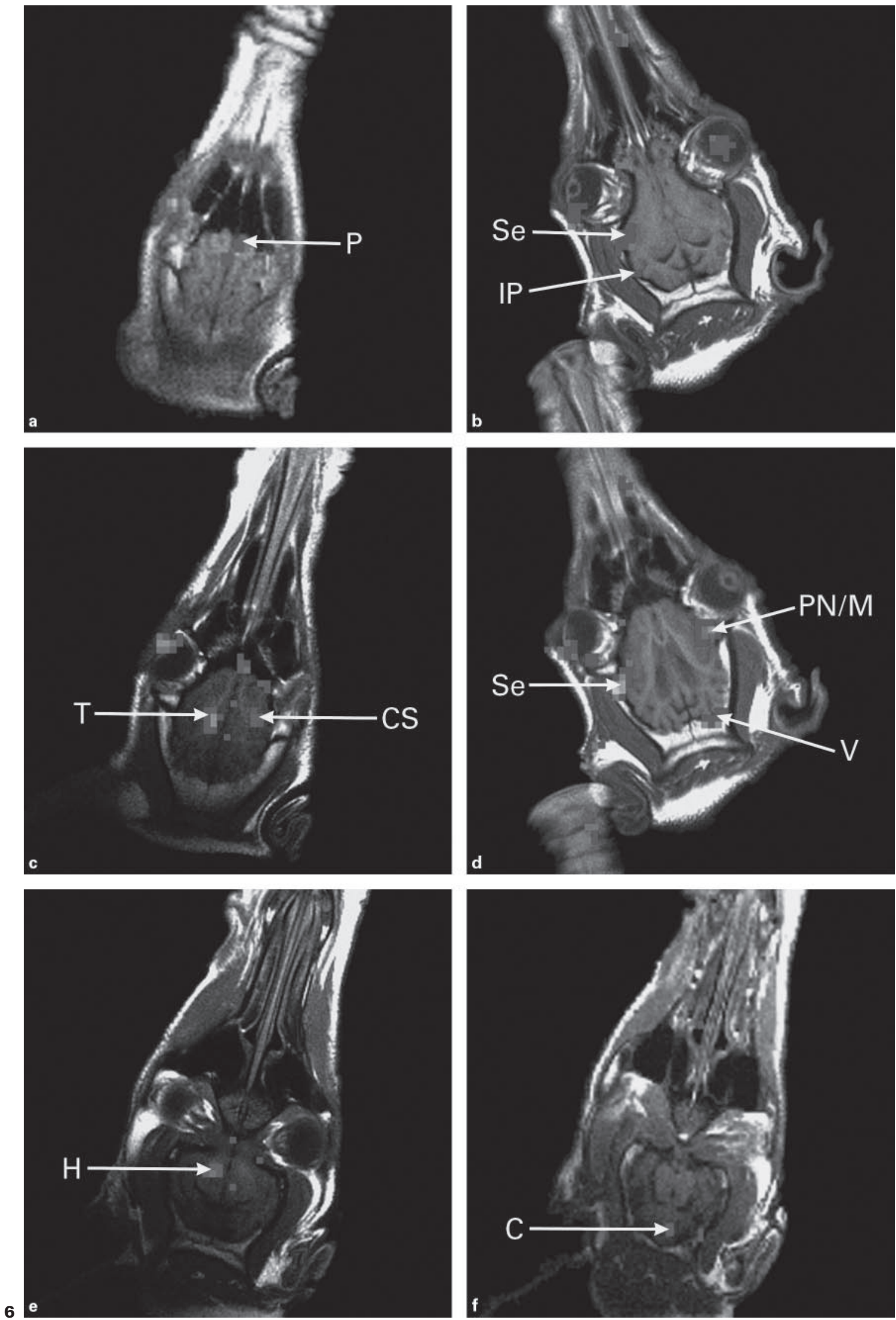

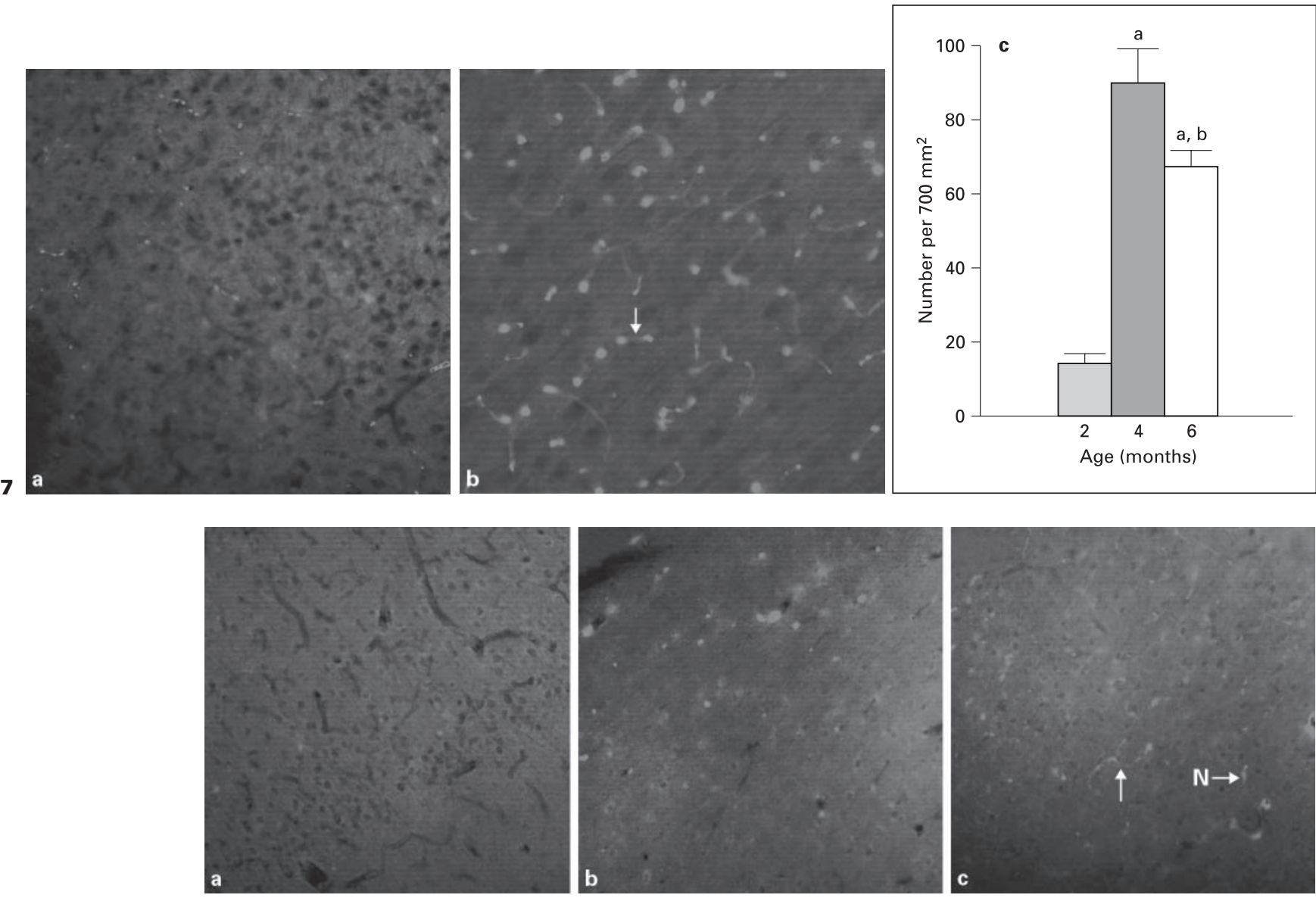

d
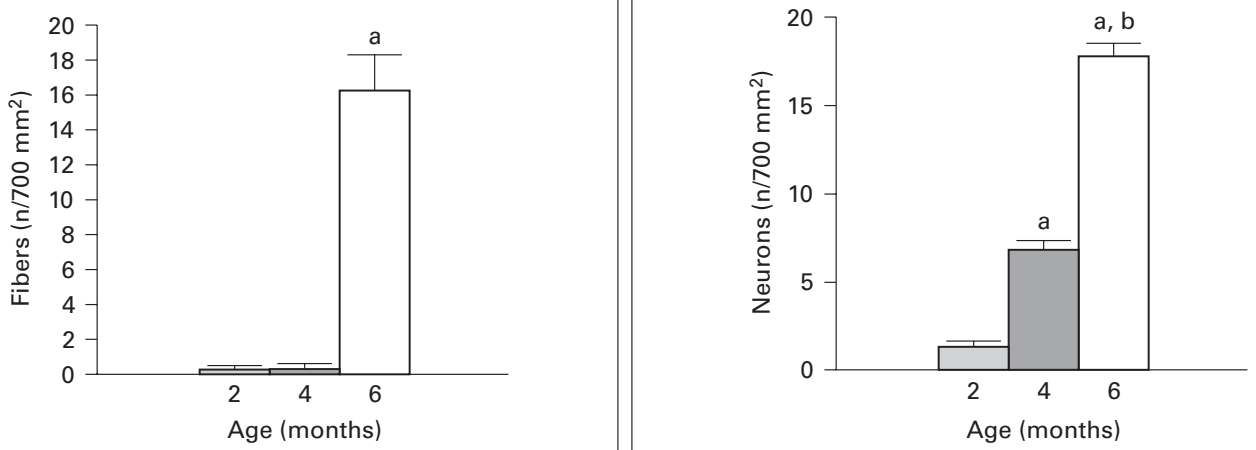

8

Fig. 7. Synaptophysin immunocytochemistry of the pig sensory cortex at 2 (a) and 6 months (b) of age $(\times 400)$ : note the increase in synaptophysin-labeled terminals at 6 months (arrow). c Numerical density of synaptophysin immunoreactive terminals/fibers in the sensory cortex. Columns represent means \pm SEM from four pigs each. ${ }^{\mathrm{a}} \mathrm{p}<0.01$ vs. 2 months, ${ }^{\mathrm{b}} \mathrm{p}<0.01$ vs. 4 months.

Fig. 8. GABA immunohistochemistry of pig sensory cortex at 2 (a), 4 (b) and 6 (c) months of age: note the increase in labeled neurons $(\mathrm{N})$ and fibers (arrow) in older animals $(\times 100)$. Numerical density of GABA-immunoreactive fibers (d) and neurons (e) in the sensory cortex. Columns represent means \pm SEM from 4 pigs each. ${ }^{a} \mathrm{p}<0.01$ vs. 2 months, ${ }^{b} \mathrm{p}<0.01$ vs. 4 months. 
cerebellum (fig. 4c). The sensory, infraparietal and temporal cortices as well as the thalamus were activated on the contralateral side (fig. $4 \mathrm{a}, \mathrm{b}$ ) by this rhythmic passive movement.

In the 4-month-old group, fMRI demonstrated an extensive but weak activation, which was predominantly located on the contralateral cortex (fig. 5a-e). Here, a response was detected in the prefrontal, motor, sensory and infraparietal cortices (fig. 5a-c). Distinct activation was also observed in the thalamus (fig. 5b) and in the septal area (fig. 5c). Bilateral activation was detected in the prefrontal cortex. Furthermore, brainstem activation was registered, involving the medial lemniscal system (fig. 5d), a major sensory pathway, on both sides and motor fiber tracts ipsilaterally (fig. 5e).

In the 6-month-old group, fMRI demonstrated activation sites which were more restricted in size but much more intense (fig. 6a-f). Again, activation was mainly observed on the contralateral side, with the sensory cortex showing strongest activation (fig. $6 \mathrm{~b}, \mathrm{~d}$ ). In addition, the infraparietal cortex (fig. 6b), the thalamus (fig. 6c) and the hypothalamus (fig. 6e) were activated on the contralateral side, whereas activation of prefrontal (fig. 6a), premotor/motor (fig. 6d) and visual (fig. 6d) cortices, corpus striatum (fig. 6c) as well as cerebellum (fig. 6f) was noted on the ipsilateral side. The relative proportion of the cortex activated in this case was $3.23 \pm 0.53 \%$ in 2 months, $21.30 \pm 4.50 \%$ in 4 months and $10.36 \pm 0.92 \%$ in 6 months. All comparisons between groups were statistically significant ( $\mathrm{p}<0.001,2$ vs. 4 months and 6 vs. 4 months, and $\mathrm{p}<0.012$ vs. 6 months).

Activations sometimes appeared outside the brain and were mainly noise signals. We therefore only described the regions with signal areas larger than 8 voxels. The eye activation should be related to eye movements, which were often uncontrollable. Besides, in the majority of cases, such activation would not trigger enough response in the visual cortex or at the most tiny activation in the visual cortex, which would not affect analysis in depth.

\section{Maturation of the Pig's Sensory Cortex}

Synaptophysin immunohistochemistry revealed relatively few labeled fibers and terminals in the sensory cortex of 2-month-old pigs (fig. 7a). By 4 months, the numerical density of immunoreactive fibers and terminals had significantly increased (fig. 7c). At the age of 6 months (fig. 7b, c), density of labeled fibers and terminals was still significantly higher than at 2 months of age. It had, however, decreased compared to 4 months of age (fig. 7c).

fMRI of Postnatal Pig Brain
GABA immunohistochemistry showed a relatively small number of GABA-positive cells and fibers in the pig's sensory cortex at the age of 2 months (fig. 8a, d, e). Until the age of 4 months, density of GABA-immunoreactive neurons increased significantly (fig. 8b, e), the number of fibers, however, remained relatively constant (fig. 8b, d). Only at the age of 6 months, a dense network of GABA-immunoreactive cells and fibers was observed (fig. 8c). At this age, density of both labeled neurons (fig. 8e) and fibers (fig. 8d) had significantly increased compared to 2 months of age.

\section{Discussion}

Our results demonstrate that fMRI studies of the brain of conscious un-anesthetized pigs are feasible without any head motion artifacts, provided the head is sufficiently immobilized. The response pattern of the pig brain has been quite uniform within each age group, and virtually no interindividual variation has been observed, which is a problem frequently encountered in human fMRI studies [18].

Our results in the oldest age group, 6-month-old pigs, show that pain stimulation results in an activation of the contralateral sensory cortex and of sensory pathways in the brainstem. Following a more complex type of stimulation, consisting of repetitive flexion and extension of the pig's hind paw by the examiner, brain activation is much more widespread involving the contralateral sensory and ipsilateral premotor and motor cortices. In addition, activation is seen in the cerebellum, the hypothalamus and septal area. Activation of the contralateral sensory cortex by passive movements is in good agreement with positron emission tomography and regional cerebral blood flow studies in humans [19,20]. Cerebellar activation has also been documented as a consequence of passive movements [21]. In humans, activation of the premotor/motor areas and of the basal ganglia is, however, typical of active movements $[19,20]$. We therefore cannot exclude that our passive movements of the pig's knee joint also elicited discrete active reflex movements.

Analysis of younger animals further demonstrates that a very simple type of sensory stimulation (e.g. pain) hardly elicits any activation in the brains of 2-month-old pigs. At 4 months, markedly increased activity is recorded in the brain, with the signals being relatively high and diffusely localized, i.e. in various cortical areas of both hemispheres. By 6 months of age, the signals obtained upon simple sensory stimulation are more discrete and largely

Neurosignals 2005;14:222-233 
confined to the contralateral sensory cortex. After the more complex repetitive passive movements, a reaction of low intensity is already obtained at 2 months of age, which is diffusely located in many regions on both sides of the brain. By 4 months, passive movement, like pain stimulation, results in a much more intense reaction, involving large brain areas bilaterally. At 6 months of age, activation is again confined to relatively few areas.

The increase in reaction intensity observed for both types of sensory stimulation between 2 and 4 months of age is most probably due to the immaturity of the pig brain at 2 months of age. Histogenesis of the pig brain is poorly documented; developmental studies mainly deal with the first 50 postnatal days and are confined to neuropeptide expression [22, 23]. Biochemical studies demonstrate that lipid and glycolipid contents of the pig's brain most rapidly increase in the first 3-4 months of postnatal development [24], indicating a growth spurt due to peak myelination $[2,24]$ and synaptogenesis. This assumption is also corroborated by our data on immunoreaction for synaptophysin, a synaptic marker [14] most strongly expressed during synapse formation [25, 26]. Density of synaptophysin-positive fibers and terminals peaks at 4 months, indicating that synaptogenesis in the pig sensory cortex only peaks around this age. fMRI activity is closely related to synaptic formation and myelination $[27,28]$. This may explain why the strongest and most widespread activation has also been observed in fMRI at 4 months.

Thereafter, at 6 months, activation becomes more restricted to specific brain regions. Neuroimaging studies of the human brain have similarly demonstrated that the magnitude of activity elicited in the neocortex is much greater and occupies a larger volume in children compared to adults [29]. The decreased focal activity pattern in children possibly reflects an immature network circuitry, with more diffuse patterns of activity being found in a relatively immature network [30]. In humans, maturation of integrated function among the neocortex, striatum, thalamus and cerebellum has been related to the ability to successfully suppress automatic behavioral responses [31].

It seems most likely that GABA, the most widespread inhibitory neurotransmitter, is involved in these inhibitory connections. GABA plays a particular role in neuronal plasticity and remodeling of synaptic connectivity during late developmental stages [15]. Our results demonstrate that, in the pig neocortex, the number of GABAimmunoreactive neurons significantly increases between 2 and 6 months and that the majority of GABAergic fibers is formed between 4 and 6 months of age. Up to now, there have been no reports on the maturation of GABAergic neurons in the pig neocortex. Our findings are, however, in good agreement with studies on the developing neocortex of other mammals, which indicate that GABAergic synapses only attain their mature distribution relatively late after birth, i.e. several months postnatally in humans and monkeys and a few weeks post partum in rodents [32]. It is therefore well conceivable that the more restricted response observed in 6-monthold pigs is related to the maturation of GABAergic neurons. Another contributing factor may be synaptic pruning and remodelling [31,33], which is also indicated by the decrease in synaptophysin immunoreaction observed between 4 and 6 months.

Comparison of fMRI responses and immunohistochemical data thus strongly suggest that the pig neocortex does not reach functional maturity before about 6 months of age.

\section{Conclusion}

Our results indicated an important trend in the functional maturation of the cortex. There was an immature stage when any small stimulus would induce global and nonspecific activities in the brain, before the establishment of discrete and specific reactive sites. This period refers to the critical stage of maturation and is of clinical importance in pediatric or veterinary pediatric neurology. This is the first study that integrated fMRI with conventional morphology to illustrate this phenomenon in an animal model.

\section{Acknowledgments}

The authors wish to thank Samuel Wong for excellent skillful technical assistance in preparing the photographs. 


\section{References}

1 Dobbing J, Sands J: Quantitative growth and development of human brain. Arch Dis Child 1973;48:757-767.

$>2$ Pond WG, Boleman SL, Fiorotto ML, Ho H, Knabe DA, Mersmann HJ, Savell JW, Su DR: Perinatal ontogeny of brain growth in the domestic pig. Proc Soc Exp Biol Med 2000;223: 102-108.

-3 Munkeby BH, Lyng K, Froen JF, WintherLarssen EH, Rosland JH, Smith HJ, Saugstad OD, Bjornerud A: Morphological and hemodynamic magnetic resonance assessment of early neonatal brain injury in a piglet model. J Magn Reson Imaging 2004;20:8-15.

4 Grate LL, Golden JA, Hoopes PJ, Hunter JV, Duhaime AC: Traumatic brain injury in piglets of different ages: techniques for lesion analysis using histology and magnetic resonance imaging. J Neurosci Methods 2003; 123:201206.

$\checkmark 5$ Martens S, Theisen A, Balzer JO, Dietrich M, Graubitz K, Scherer M, Schmitz C, Doss M, Moritz A: Improved cerebral protection through replacement of residual intracavital air by carbon dioxide: a porcine model using diffusion-weighted magnetic resonance imaging. J Thorac Cardiovasc Surg 2004;127:5156.

6 McDannold N, King RL, Hynynen K: MRI monitoring of heating produced by ultrasound absorption in the skull: in vivo study in pigs. Magn Reson Med 2004;51:1061-1065.

$\checkmark 7$ Duhaime AC, Hunter JV, Grate LL, Kim A, Golden J, Demidenko E, Harris C: Magnetic resonance imaging studies of age-dependent responses to scaled focal brain injury in the piglet. J Neurosurg 2003;99:542-548.

-8 Peeters-Scholte C, Braun K, Koster J, Kops N, Blomgren K, Buonocore G, van Buul-Offers S, Hagberg H, Nicolay K, van Bel F, Groenendaal F: Effects of allopurinol and deferoxamine on reperfusion injury of the brain in newborn piglets after neonatal hypoxia-ischemia. Pediatr Res 2003;54:516-522.

$\checkmark 9$ Marcilloux JC, Felix MB, Rampin O, Stoffels C, Ibazizen MT, Cabanis EA, Laplace JP, Albe-Fessard D: Preliminary results of a magnetic resonance imaging (MRI) study of the pig brain placed in stereotaxic conditions. Neurosci Lett 1993;156:113-116.
10 Watanabe H, Andersen F, Simonsen CZ, Evans SM, Gjedde A, Cumming P, DaNex Study Group: MR-based statistical atlas of the Göttingen minipig brain. Neuroimage 2001;14: 1089-1096.

11 Rivkin MJ: Developmental neuroimaging of children using magnetic resonance techniques. Ment Retard Dev Disabil Res Rev 2000;6:6880.

12 Makiranta MJ, Jauhiainen JP, Oikarinen JT, Suominen K, Tervonen O, Alahuhta S, Jantti $\mathrm{V}$ : Functional magnetic resonance imaging of swine brain during change in thiopental anesthesia into EEG burst-suppression level - a preliminary study. MAGMA 2002;15:27-35.

13 Saito T: Functional study of the central nervous system for farm animal husbandry. Anim Sci J 2002;73:77-82.

14 Valtorta F, Pennuto M, Bonanomi D, Benfenati F: Synaptophysin: leading actor or walkon role in synaptic vesicle exocytosis? Bioessays 2004;26:445-453.

15 Möhler H, Fritschy JM, Crestani F, Hensch T, Rudolph U: Specific GABA(A) circuits in brain development and therapy. Biochem Pharmacol 2004;68:1685-1690.

-16 Felix B, Leger M, Albe-Fessard D, Marcilloux JC, Rampin O, Laplace JP: Stereotaxic atlas of the pig brain. Brain Res Bull 1999;49:1-137.

17 Hedreen JC: What was wrong with the Abercrombie and empirical cell counting methods? A review. Anat Rec 1998;250:373-380.

18 Santosh PJ: Neuroimaging in child and adolescent psychiatric disorders. Arch Dis Child 2000;82:412-419.

19 Weiller C, Juptner M, Fellows S, Rijntjes M, Leonhardt G, Kiebel S, Muller S, Diener HC, Thilmann AF: Brain representation of active and passive movements. Neuroimage 1996;4: 105-110.

20 Mima T, Sadato N, Yazawa S, Hanakawa T, Fukuyama H, Yonekura Y, Shibasaki H: Brain structures related to active and passive finger movements in man. Brain 1999;122:19891997.

21 Jueptner M, Ottinger S, Fellows SJ, Adamschewski J, Flerich L, Muller SP, Diener HC, Thilmann AF, Weiller C: The relevance of sensory input for the cerebellar control of movements. Neuroimage 1997;5:41-48.

$\checkmark 22$ Pearson PL, Anderson LL, Jacobsen CD: The prepubertal ontogeny of galanin-like immunoreactivity in the male Meishan pig brain. Brain Res Dev Brain Res 1996;92:125-139.
23 Pearson PL, Anderson LL, Jacobson CD: The prepubertal ontogeny of neuropeptide Y-like immunoreactivity in the male Meishan pig brain. Brain Res Dev Brain Res 1996;91:4169.

24 Flynn TJ: Developmental changes of myelinrelated lipids in brain of miniature swine. Neurochem Res 1984;9:935-945.

-25 Leclerc N, Beesley PW, Brown I, Colonnier M, Gurd JW, Paladino T, Hawkes R: Synaptophysin expression during synaptogenesis in the rat cerebellar cortex. J Comp Neurol 1989;280: 197-212.

-26 Sarnat HB, Born DE: Synaptophysin immunocytochemistry with thermal intensification: a marker of terminal axonal maturation in the human fetal nervous system. Brain Dev 1999; 21:41-50

27 Yamada H, Sadato N, Konishi Y, Kimura K, Tanaka M, Yonekura Y, Ishii Y: A rapid brain metabolic change in infants detected by fMRI. Neuroreport 1997;8:3775-3778.

-28 Yamada H, Sadato N, Konishi Y, Muramoto S, Kimura K, Tanaka M, Yonekura Y, Ishii Y, Itoh $\mathrm{H}$ : A milestone for normal development of the infantile brain detected by functional MRI. Neurology 2000;55:218-223.

29 Casey BJ, Giedd JN, Thomas KM: Structural and functional brain development and its relation to cognitive development. Biol Psychol 2000;54:241-257.

30 Davidson MC, Thomas KM, Casey BJ: Imaging the developing brain with fMRI. Ment Retard Dev Disabil Res Rev 2003;9:161-167.

- 31 Luna B, Thulborn KR, Munoz DP, Merriam EP, Garver KE, Minshew NJ, Keshavan MS, Genovese CR, Eddy WF, Sweeney JA: Maturation of widely distributed brain function subserves cognitive development. Neuroimage 2001;13:786-793.

32 Conti F, Minelli A, Melone M: GABA transporters in the mammalian cerebral cortex: localization, development and pathological implications. Brain Res Brain Res Rev 2004;45: 196-212.

33 Agmon A, Hollrigel G, O’Dowd DK: Functional GABAergic synaptic connection in neonatal mouse barrel cortex. J Neurosci 1996;16: 4684-4695. 